\section{Comunidades quilombolas de Vitória da Conquista, Bahia, Brasil: hipertensão arterial e fatores associados}

\author{
Quilombo communities in Vitória da Conquista, \\ Bahia State, Brazil: hypertension and associated \\ factors
}

\section{Comunidades quilombolas de Vitória da Conquista, Bahía, Brasil: hipertensión y factores asociados}

\author{
${ }^{1}$ Instituto Multidisciplinar \\ em Saúde, Universidade \\ Federal da Bahia, Vitória da \\ Conquista, Brasil. \\ 2 Faculdade de Medicina, \\ Universidade Federal de \\ Minas Gerais, Belo Horizonte, \\ Brasil. \\ 3 Instituto de Ciências Exatas, \\ Universidade Federal de \\ Minas Gerais, Belo Horizonte, \\ Brasil. \\ Correspondência \\ V. M. Bezerra \\ Instituto Multidisciplinar em \\ Saúde, Universidade Federal \\ da Bahia. \\ Rua Rio de Contas 58 , \\ Quadra 17, Lote 58, Campus \\ Anísio Teixeira, Vitória da \\ Conquista, BA 45029-094, \\ Brasil. \\ vanessaenut@yahoo.com.br
}

\begin{abstract}
The study aimed to estimate the prevalence of hypertension in members of quilombos (slavedescendant African-Brazilian communities) and to evaluate possible associated factors. A population-based survey included 797 individuals 18 years or older. Hypertension was defined as systolic blood pressure $\geq 140 \mathrm{mmHg}$ or diastolic blood pressure $\geq 90 \mathrm{mmHg}$ or reported use of antihypertensive medication. We used Poisson regression with robust variance and hierarchical entry of variables. Prevalence of hypertension was 45.4\% (95\%CI: 41.89-48.85). Factors associated with hypertension were: neighborhood security (distal), age, economic class, education, and physical inactivity (intermediate), and body mass index (proximal). Health promotion is needed in the quilombo communities through action at the individual and population levels. The high prevalence of hypertension highlights the need for broad access to health services for prevention, early diagnosis, and guidelines for adequate management.
\end{abstract}

Hypertension; Risk Factors; Vulnerable Groups; African Continental Ancestry Group; Cross-sectional Studies
Vanessa Moraes Bezerra 1,2

Amanda Cristina de Souza Andrade 2

Cibele Comini César 2,3

Waleska Teixeira Caiaffa 2

\section{Resumo}

Este estudo objetivou estimar a prevalência de hipertensão arterial em residentes das comunidades quilombolas e avaliar possíveis fatores associados. Foi realizado um inquérito de base populacional em uma amostra de 797 indivíduos com 18 anos ou mais. A hipertensão arterial foi definida por pressão arterial sistólica $\geq 140 \mathrm{mmHg}$ elou pressão arterial diastólica $\geq$ $90 \mathrm{mmHg}$ elou uso de medicamentos anti-hipertensivos. Empregou-se a regressão de Poisson com variância robusta, adotando a entrada hierárquica de variáveis. A prevalência de hipertensão foi de 45,4\% (IC95\%: 41,89-48,85), o fator distal: segurança na vizinhança; os fatores intermediários: idade, classe econômica, escolaridade e inatividade física; e o fator proximal: índice de massa corporal mostraram-se associados com a hipertensão. Observa-se a necessidade da promoção da saúde por meio de atenção inclusiva aos quilombolas, valendo-se de ações em níveis individual e populacional. A alta prevalência de hipertensão arterial reforça a necessidade de um amplo acesso aos serviços de saúde para prevenção, diagnóstico precoce e orientações para o manejo adequado.

Hipertensão; Fatores de Risco; Comunidades Vulneráveis; Grupo com Ancestrais do Continente Africano; Estudos Transversais 


\section{Introdução}

A hipertensão arterial é a principal causa de óbitos evitáveis nos países em desenvolvimento e no mundo ${ }^{1}$, sendo considerada um fator de risco modificável para doenças cardiovasculares e um dos mais importantes problemas de saúde pública, por sua magnitude, risco e dificuldades no seu controle ${ }^{2}$. A hipertensão também representa um dos principais problemas de saúde pública nas populações negras em todo o mundo ${ }^{3} \mathrm{e}$, no Brasil, por ser mais frequente em populações afrodescendentes, destaca-se no contexto das doenças mais importantes por razões étnicas 4 .

Estudos de base populacional realizados nos últimos 10 anos demonstram que a prevalência de hipertensão arterial, na população brasileira com 18 anos ou mais, varia de $18,9 \%$ a $36,5 \%$, dependendo do grupo estudado, da região ou da definição adotada 5,6,7,8. A etnia, assim como idade, sexo, características socioeconômicas, consumo de sal, obesidade, consumo de álcool e a inatividade física, são fatores importantes de risco para o desenvolvimento de hipertensão arterial 9. Apesar de conhecidos os fatores de risco, inquéritos domiciliares recentes revelaram baixos níveis (20\% a 39\%) de controle da pressão arterial 10,11.

O Brasil é a segunda maior nação negra do mundo, atrás somente da Nigéria. Segundo dados do Instituto Brasileiro de Geografia e Estatística (IBGE) de 2010, a população de pretos e pardos no Brasil corresponde a 50,7\% dos habitantes 12 . Dada a persistência de disparidades raciais, evidências sistemáticas indicam que os negros têm maior incidência de doenças e morrem mais precocemente, em todas as idades 13 . Dentre as doenças que acometem essa população, a hipertensão arterial aparece em destaque, sendo cerca de duas vezes mais prevalente em indivíduos de cor não branca 14,15 e fortemente associada a estratos sociais mais baixos 3,14. Ao se contextualizar a saúde da população negra é necessário considerar as condições especiais de vulnerabilidade vivenciadas por este grupo.

No tocante às desigualdades étnico-raciais, a situação dos grupos quilombolas merece destaque por se tratar de um grupo em situação de vulnerabilidade social, também decorrente de um processo histórico de expropriação de cultura e de direitos, cujos impactos tiveram reflexo nos indicadores de saúde desta população 16,17 . A definição de comunidades quilombolas está baseada na autoatribuição, com trajetória histórica própria, dotadas de relações territoriais específicas e com presunção de ancestralidade negra relacionada com a resistência à opressão histórica sofrida 18.
Na literatura disponível não existem estudos epidemiológicos que caracterizem a situação de saúde das populações quilombolas, particularmente no sudoeste do Estado da Bahia, região do entorno de Vitória da Conquista, que permitam o delineamento para implementação e acompanhamento do impacto de ações e políticas voltadas para a melhoria de suas condições de vida e saúde.

A relevância de um recorte étnico-racial relativo às doenças e às condições de vida da população quilombola permite que sejam identificados contingentes populacionais mais suscetíveis a agravos à saúde, como a hipertensão. E por serem os negros a maioria dos socialmente excluídos, o quilombo vem a ser, portanto, o mote principal para se discutir uma parte da cidadania negada 19.

Diante da magnitude que representa a hipertensão, entende-se que todos os esforços devem ser feitos no sentido de viabilizar estudos que sejam direcionados para o conhecimento deste agravo em grupos populacionais específicos. O objetivo do presente estudo foi identificar a prevalência de hipertensão e investigar fatores associados em comunidades quilombolas do Município de Vitória da Conquista.

\section{Métodos}

\section{Desenho e amostra do estudo}

Trata-se de um estudo de corte transversal que faz parte do projeto de pesquisa COMQUISTA - Comunidades Quilombolas de Vitória da Conquista: Avaliação de Saúde e Seus Condicionantes ${ }^{20}$, realizado no período de setembro a de outubro de 2011. A população consistiu de indivíduos com 18 anos ou mais, residentes em comunidades quilombolas do município.

O plano amostral foi obtido valendo-se da estimativa populacional baseada no Consolidado da Situação de Comunidades Quilombolas de Vitória da Conquista (dados primários, Consolidado da Situação de Comunidades Quilombolas de Vitória da Conquista - Bahia, 2010), cedida pela Secretaria Municipal de Saúde. Vitória da Conquista registra 25 comunidades quilombolas certificadas pela Fundação Palmares, sediadas em cinco distritos da região. Os critérios de inclusão adotados foram possuir certificação pela Fundação Palmares e ter mais de 50 famílias na comunidade.

A população de pesquisa foi estimada em 2.935 indivíduos adultos oriundos de $10 \mathrm{comu}$ nidades quilombolas. Para o cálculo do tamanho amostral foi considerada uma prevalência de 
$50 \%$, dado a heterogeneidade dos eventos mensurados no projeto principal, precisão de $5 \%$, intervalo de $95 \%$ de confiança (IC95\%), efeito de desenho $=2$, e $30 \%$ de perdas, totalizando uma amostra de 884 indivíduos adultos.

O delineamento amostral foi realizado em dois estágios: (1) Seleção aleatória de uma comunidade quilombola em cada distrito, com probabilidade proporcional à população da comunidade. Quando o distrito tinha somente uma comunidade, esta foi selecionada; (2) Seleção aleatória dos domicílios de acordo com a distribuição proporcional de domicílios por distrito. Todos os residentes com 18 anos ou mais dos domicílios selecionados foram convidados a participar da pesquisa.

\section{Coleta dos dados}

Os dados foram coletados por meio de entrevistas individuais, realizadas nos domicílios selecionados por entrevistadores capacitados pela equipe coordenadora do projeto, utilizando um computador portátil (HP Pocket Rx5710, Hewlett-Packard Development Company, Estados Unidos). O instrumento de entrevistas utilizado foi o questionário semiestruturado da Pesquisa Nacional de Saúde (PNS) 21, em versão adaptada para a população quilombola.

Além das entrevistas, foram realizadas a aferição da pressão arterial e medidas antropométricas. Para verificar a dinâmica de recrutamento, testar os instrumentos de coleta de dados e confirmar a viabilidade da investigação foi realizado um estudo piloto em uma comunidade quilombola não selecionada para a pesquisa.

Foram realizadas três medidas de pressão arterial (com intervalo de um minuto entre cada aferição) sendo utilizado o esfigmomanômetro digital da marca Omron modelo HEM-742 (OMRON Corp., São Paulo, Brasil), validado internacionalmente 22 . As aferições foram feitas ao final da entrevista visando a garantir que os indivíduos estivessem em repouso, sentados, com as pernas descruzadas, os pés apoiados no chão, o dorso recostado na cadeira e relaxado, com o braço esquerdo apoiado sobre a mesa à altura do coração. Certificou-se de que os mesmos: não estavam com a bexiga cheia, não praticaram exercícios físicos há pelo menos 60 minutos, não ingeriram bebidas alcoólicas, café ou alimento e não fumaram nos 30 minutos anteriores ${ }^{9}$.

O peso foi verificado com os indivíduos descalços e vestindo roupas leves, em balança eletrônica portátil da marca Marte (modelo LC200pp, Marte, São Paulo, Brasil) com capacidade para $200 \mathrm{~kg}$ e sensibilidade de $50 \mathrm{~g}$. Para a aferição da altura foi utilizado o estadiômetro portátil da marca CauMaq (modelo est-22, CauMaq Indústria Metalúrgica Ltda., Cachoeira do Sul, Brasil) com os indivíduos descalços e em posição ereta. A circunferência da cintura foi medida por meio de uma fita métrica inelástica da marca Cardiomed com precisão de $0,1 \mathrm{~cm}$. A fita foi posicionada na cintura natural, ponto médio entre o último arco costal e a crista ilíaca.

\section{Variáveis estudadas}

A variável dependente do estudo foi a hipertensão. Para análise foi realizada a média das duas últimas aferições, sendo considerados hipertensos os indivíduos que apresentaram pressão arterial sistólica $\geq 140 \mathrm{mmHg}$ e/ou pressão arterial diastólica $\geq 90 \mathrm{mmHg}$ e/ou relataram uso de medicamentos anti-hipertensivos 23 .

As variáveis independentes foram estabelecidas utilizando-se um modelo conceitual para hipertensão (Figura 1), adaptado do modelo proposto por Diez Roux 24 para doenças cardiovasculares, sendo organizadas em quatro blocos. Para o primeiro bloco foram consideradas as variáveis relacionadas ao entorno físico e social, sendo questionados os aspectos relativos à vizinhança: presença de área de lazer, estética do lugar (presença de lixo ou entulho), disponibilidade de alimentos saudáveis (local para comprar frutas e verduras), presença de ruído (barulho na vizinhança) e foi questionado se o indivíduo se sente seguro ao caminhar na vizinhança durante dia e noite (variável segurança).

Variáveis sociodemográficas (escolaridade, ocupação, classe econômica, sexo, idade, estado conjugal e cor da pele) constituíram o segundo bloco, a idade foi categorizada em: 18-29, 30-39, 40-49, 50-59 e 60 anos ou mais, e estado conjugal: casado, nunca foi casado, separado/divorciado e viúvo. A escolaridade foi categorizada em: nunca estudou, 1-4 e 5 ou mais anos de estudos. Em relação à ocupação foi questionado aos participantes qual é ou era sua principal ocupação no trabalho, as repostas foram categorizadas em: nunca trabalhou, lavrador, empregado doméstico e outras ocupações. Como proxy de padrões sociais os indivíduos foram classificados em classes econômicas, de acordo com o critério da Associação Brasileira de Empresas de Pesquisas e Mercados (ABEP. Critérios de classificação econômica no Brasil. http://www.abep.org/novo/filegenerate. ashx?id=257, acessado em 21/Mar/2012).

O terceiro bloco foi constituído por variáveis de estilo de vida (uso de fumo, consumo de álcool, dieta, inatividade física, realização de atividades em grupo e estresse). Em relação ao hábito de fumar foram adotadas três categorias de classificação: nunca fumou, fumante e ex-fumante. 


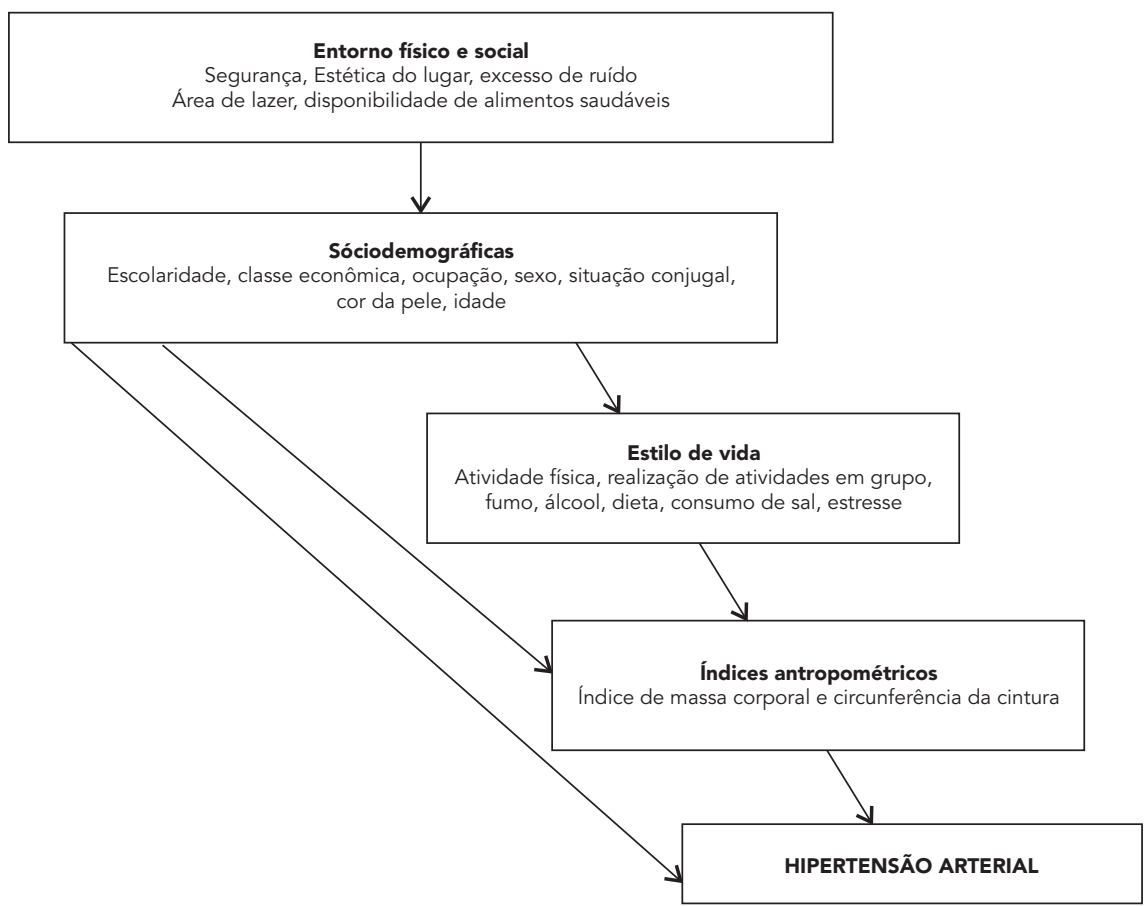

Fonte: adaptado de Diez Roux 24.

O consumo de álcool foi expresso em gramas de etanol/dia e definido como: risco, o consumo diário médio acima $30 \mathrm{~g} /$ dia (mais de duas doses padronizadas de bebidas para homens) e acima de 15g/dia (mais de uma dose padronizada para mulheres) 25 .

O consumo diário de legumes/verduras, frutas e sal foram utilizados para avaliar a dieta. $\mathrm{O}$ consumo de sal foi estimado pela quantidade consumida mensalmente; este dado foi corrigido pelo número de pessoas que realizavam as refeições no domicílio 26. Para estimar a frequência diária do consumo de frutas e legumes/verduras foram combinadas duas perguntas (número de vezes que consome na semana e número de vezes que consome no dia). Devido ao baixo número de pessoas que relataram consumir mais que 3 vezes no dia de acordo com as recomendações do Guia Alimentar para População Brasileira 27, as variáveis foram dicotomizadas em: não consome e consome pelo menos uma vez ao dia.

Foram considerados como inativos os indivíduos que praticavam menos 150 minutos de atividade física semanal 28 , em cada um dos domínios avaliados (lazer, deslocamento, trabalho e doméstico). Para a variável "atividades em grupo" foi relatada a frequência de atividades esportivas ou artísticas em grupo nos últimos 12 meses. A variável "problemas no sono nos últimos 15 dias" foi utilizada como proxy do estresse, sendo categorizada em: nunca, de vez em quando, boa parte do tempo/a maior parte do tempo.

Índices antropométricos (índice de massa corporal - IMC, circunferência da cintura - CC) compuseram o quarto bloco. Para a categorização do IMC $\left(\mathrm{kg} / \mathrm{m}^{2}\right)$ foram utilizados os pontos de corte para adultos 29: $<18,5$ (baixo peso), $\geq$ 18,5 e $<25$ (eutrófico), $\geq 25$ e $<30$ (sobrepeso) e $\geq 30$ (obesidade); e idosos 30: $\leq 22$ (baixo peso), $>22$ e $<27$ (eutrófico) e $\geq 27$ (sobrepeso). A circunferência da cintura foi considerada aumentada quando: $\geq 94 \mathrm{~cm}$ para homens $\mathrm{e} \geq 80 \mathrm{~cm}$ para mulheres, e muito aumentada $\geq 102 \mathrm{~cm} \mathrm{e} \geq 88 \mathrm{~cm}$ para homens e mulheres, respectivamente 29 . 


\section{Análise estatística}

Inicialmente, foi feita uma estimativa da prevalência de hipertensão entre os adultos residentes nas comunidades quilombolas com IC95\%. Para verificar os fatores associados à hipertensão, realizou-se análise univariada com estimativas de razões de prevalência com cálculo dos respectivos intervalos de confiança. Empregou-se a regressão de Poisson com variância robusta, sendo selecionadas as variáveis que apresentaram significância estatística de $20 \%(\mathrm{p}<0,20)$ para serem inseridas no modelo multivariado.

Foi adotada a entrada hierárquica 31 das variáveis em blocos, de acordo com a seguinte ordem: variáveis do entorno físico e social; variáveis sociodemográficas; variáveis de estilo de vida e índices antropométricos. As variáveis dos blocos mais distais permaneceram como fatores de ajuste para as os blocos hierarquicamente inferiores. Para a interpretação dos resultados, a identificação de associação estatisticamente significativa $(\mathrm{p}<0,05)$ entre um determinado fator em estudo e a hipertensão após ajuste para os potenciais fatores do mesmo bloco e dos blocos hierárquicos superiores indicam a existência de um efeito independente, próprio do referido fator. A comparação entre modelos foi feita pelo critério de Akaike (AIC).

Uma vez que todos os indivíduos com 18 anos ou mais do domicílio foram entrevistados, foi avaliado o efeito de agrupamento intradomiciliar utilizando-se a estatística DEFT (design effect), e como não foi evidenciado efeito de cluster, optou-se pelo modelo que não considera o efeito de agrupamento.

Todas as análises foram realizadas no programa Stata versão 10.0 (Stata Corp., College Station, Estados Unidos).

\section{Questões éticas}

O projeto foi aprovado pelos Comitês de Ética em Pesquisa da Faculdade São Francisco de Barreiras (CAAE 0118.0.066.000-10) e da Universidade Federal de Minas Gerais (CAAE 0118.0.066.203-10), em consonância com o disposto na Resolução no 196/96 do Conselho Nacional de Saúde. Os participantes foram informados sobre os objetivos do trabalho, procedimentos e sigilo dos dados, e manifestaram sua concordância em participar da pesquisa ao assinar o Termo de Consentimento Livre Esclarecido.

\section{Resultados}

Nos 422 domicílios visitados foram identificados 943 indivíduos elegíveis para o estudo e, destes, 797 participaram da pesquisa. A perda de 15,5\% da amostra foi devida, principalmente, por não terem sido encontrados os indivíduos selecionados após três visitas ao seu domicílio $(82,2 \%)$. Ao compararmos os grupos de participantes com o de não participantes, a perda foi significativamente maior em indivíduos do sexo masculino e com idades entre 18 a 34 anos. Contudo, ao realizar a ponderação das perdas diferenciais usando fatores de calibração, não foram observadas diferenças significativas nas estimativas de ponto da prevalência de hipertensão. Desse modo, as análises foram conduzidas sem considerar a ponderação.

Dos 797 indivíduos que aceitaram participar do projeto COMQUISTA, foram excluídos 8 participantes $(1,0 \%)$ devido à presença de dados faltantes em alguma das variáveis utilizadas para classificação da hipertensão arterial. A prevalência de hipertensão encontrada nessa população estudada foi de 45,4\% (IC95\%: 41,9-48,8).

A maioria tinha entre 18 e 29 anos $(25,2 \%)$ com idade média de $44 \pm 17,9$ anos, era do sexo feminino $(54,3 \%)$, casados $(61,4 \%)$ e de cor não branca (87,3\%). Mais de $80 \%$ da população foram classificados nas categorias D e E para as classes econômicas e apenas $27,6 \%$ estudaram por pelo menos 5 anos. A principal ocupação relatada foi a de lavrador seguida de empregado doméstico. Com exceção de sexo e cor, todas as variáveis sociodemográficas apresentaram associações significativas com a hipertensão arterial. Foram observadas maiores prevalências de hipertensão arterial em indivíduos com 60 anos ou mais, viúvos, que nunca estudaram e inseridos em classes econômicas mais baixas. A ocupação de lavrador resultou na maior prevalência (61\%) de hipertensão arterial (Tabela 1).

Quanto às características da vizinhança, $21,6 \%$ relataram a presença de lixo, $73,6 \%$ de ausência de área de lazer, 32,6\% de ausência de segurança, cerca de $80 \%$ de indisponibilidade de locais para comprar frutas e verduras e ausência de barulho (81,9\%). Entre essas variáveis, segurança na vizinhança apresentou associação significativa com hipertensão arterial (Tabela 1).

Hábito de fumar no momento do estudo foi relatado por $19,6 \%$ e o consumo de álcool considerado de risco em $13,1 \%$. O grupo de indivíduos classificados como ex-fumantes apresentou maior razão de prevalência de hipertensão arterial em relação aos que nunca fumaram, e o consumo de álcool foi associado negativamente à hipertensão (Tabela 2). 
Tabela 1

Distribuição da hipertensão em relação às variáveis sociodemográficas e do entorno físico e social. Projeto COMQUISTA, Brasil, 2011.

\begin{tabular}{|c|c|c|c|c|c|}
\hline Variável & n (\%) & $\begin{array}{c}\text { Prevalência de hipertensão } \\
\text { arterial (\%) }\end{array}$ & $\mathrm{RP}$ & IC95\% & Valor de $p$ \\
\hline \multicolumn{6}{|l|}{ Sexo } \\
\hline Masculino & $364(45,7)$ & 44,4 & 1,00 & & 0,632 \\
\hline Feminino & $433(54,3)$ & 46,1 & 1,04 & $0,89-1,21$ & \\
\hline \multicolumn{6}{|l|}{ Idade (anos) } \\
\hline $18-29$ & $201(25,2)$ & 15,1 & 1,00 & & $<0,001$ \\
\hline $30-39$ & $166(20,8)$ & 32,3 & 2,14 & $1,44-3,19$ & \\
\hline $40-49$ & $149(18,7)$ & 45,9 & 3,04 & $2,09-4,42$ & \\
\hline $50-59$ & $104(13,1)$ & 63,5 & 4,21 & $2,93-6,04$ & \\
\hline 60 ou mais & $177(22,2)$ & 80,7 & 5,35 & $3,82-7,50$ & \\
\hline \multicolumn{6}{|l|}{ Estado conjugal } \\
\hline Casado/Em união & $489(61,4)$ & 47,5 & 1,00 & & $<0,001$ \\
\hline Nunca foi casado & $187(23,5)$ & 25,5 & 0,54 & $0,41-0,70$ & \\
\hline Separado/Divorciado/Viúvo & $121(15,2)$ & 67,2 & 1,41 & $1,21-1,65$ & \\
\hline \multicolumn{6}{|l|}{ Cor da pele } \\
\hline Branca & $100(12,7)$ & 44,3 & 1,00 & & 0,856 \\
\hline Não branca & $687(87,3)$ & 45,3 & 1,02 & $0,81-1,30$ & \\
\hline \multicolumn{6}{|l|}{ Classe econômica } \\
\hline $\mathrm{Ce} B 2$ & $114(14,4)$ & 35,1 & 1,00 & & 0,003 \\
\hline $\mathrm{D}$ & $401(50,8)$ & 43,3 & 1,23 & $0,94-1,62$ & \\
\hline E & $275(34,8)$ & 53,1 & 1,51 & $1,15-1,99$ & \\
\hline \multicolumn{6}{|l|}{ Escolaridade (anos) } \\
\hline 5 ou mais & $219(27,7)$ & 16,5 & 1,00 & & $<0,001$ \\
\hline $1-4$ & $299(37,7)$ & 47,7 & 2,88 & $2,09-3,98$ & \\
\hline Nunca estudou & $274(34,6)$ & 66,0 & 4,00 & $2,93-5,46$ & \\
\hline \multicolumn{6}{|l|}{ Principal ocupação } \\
\hline Outras ocupações & $172(21,6)$ & 33,7 & 1,00 & & $<0,001$ \\
\hline Lavrador(a) & $413(51,8)$ & 54,3 & 1,61 & $1,28-2,02$ & \\
\hline Doméstico(a) & $113(14,2)$ & 37,5 & 1,11 & $0,81-1,53$ & \\
\hline Nunca trabalhou & $99(12,4)$ & 37,5 & 1,11 & $0,79-1,55$ & \\
\hline \multicolumn{6}{|l|}{ Lixo na vizinhança } \\
\hline Não & $619(78,4)$ & 45,8 & 1,00 & & 0,695 \\
\hline Sim & $171(21,6)$ & 44,1 & 0,96 & $0,79-1,16$ & \\
\hline \multicolumn{6}{|l|}{ Área de lazer } \\
\hline $\operatorname{Sim}$ & $209(26,4)$ & 41,5 & 1,00 & & 0,198 \\
\hline Não & $582(73,6)$ & 46,9 & 1,13 & $0,94-1,35$ & \\
\hline \multicolumn{6}{|l|}{$\begin{array}{l}\text { Local para comprar frutas/ } \\
\text { verduras }\end{array}$} \\
\hline Sim & $145(18,3)$ & 42,4 & 1,00 & & 0,418 \\
\hline Não & $646(81,7)$ & 46,2 & 1,09 & $0,88-1,34$ & \\
\hline \multicolumn{6}{|l|}{ Segurança na vizinhança } \\
\hline $\operatorname{Sim}$ & $532(67,3)$ & 42,4 & 1,00 & & 0,013 \\
\hline Não & $258(32,6)$ & 51,6 & 1,22 & $1,04-1,42$ & \\
\hline \multicolumn{6}{|l|}{ Barulho na vizinhança } \\
\hline Não & $648(81,9)$ & 45,2 & 1,00 & & 0,787 \\
\hline Sim & $143(18,1)$ & 46,5 & 1,03 & $0,84-1,25$ & \\
\hline
\end{tabular}

IC95\%: intervalo de 95\% de confiança; RP: razão de prevalência. 
Distribuição da hipertensão em relação às variáveis estilo de vida e índices antropométricos. Projeto COMQUISTA, Brasil, 2011

\begin{tabular}{|c|c|c|c|c|c|}
\hline Variável & n (\%) & $\begin{array}{c}\text { Prevalência de hipertensão } \\
\text { arterial (\%) }\end{array}$ & RP & IC95\% & Valor de $p$ \\
\hline \multicolumn{6}{|l|}{ Fumo } \\
\hline Nunca fumou & $431(54,1)$ & 39,2 & 1,00 & & $<0,001$ \\
\hline Fumante & $156(19,6)$ & 47,1 & 1,20 & $0,98-1,47$ & \\
\hline Ex-fumante & $210(26,3)$ & 56,7 & 1,45 & $1,22-1,71$ & \\
\hline \multicolumn{6}{|l|}{ Álcool (consumo de risco) } \\
\hline Não & $688(86,9)$ & 46,7 & 1,00 & & 0,049 \\
\hline Sim & $104(13,1)$ & 35,6 & 0,76 & $0,58-0,99$ & \\
\hline \multicolumn{6}{|l|}{ Consumo de legumes/verduras } \\
\hline Consome * & $669(84,6)$ & 43,7 & 1,00 & & 0,018 \\
\hline Não consome & $122(15,4)$ & 54,6 & 1,25 & $1,04-1,50$ & \\
\hline \multicolumn{6}{|l|}{ Consumo de frutas } \\
\hline Consome * & $635(80,6)$ & 43,6 & 1,00 & & 0,088 \\
\hline Não consome & $153(19,4)$ & 51,0 & 1,17 & $0,98-1,40$ & \\
\hline \multicolumn{6}{|l|}{ Consumo de sal (g/dia) } \\
\hline$\leq 5,0$ & $293(40,1)$ & 39,7 & 1,00 & & 0,074 \\
\hline $5,1-8,4$ & $224(30,7)$ & 47,3 & 1,19 & $0,98-1,45$ & \\
\hline$\geq 8,5$ & $213(29,2)$ & 49,3 & 1,24 & $1,02-1,51$ & \\
\hline \multicolumn{6}{|l|}{ Problemas com o sono } \\
\hline Nunca & $376(47,3)$ & 45,2 & 1,00 & & 0,065 \\
\hline De vez em quando & $260(32,7)$ & 41,1 & 0,91 & $0,76-1,09$ & \\
\hline Bom/a maior parte do tempo & $159(20,0)$ & 52,5 & 1,16 & $0,96-1,40$ & \\
\hline \multicolumn{6}{|l|}{ Categorias de IMC ** } \\
\hline Baixo peso/Eutrófico & $484(63,1)$ & 41,7 & 1,00 & & 0,016 \\
\hline Sobrepeso & $234(30,5)$ & 50,9 & 1,22 & $1,03-1,44$ & \\
\hline Obeso & $49(6,4)$ & 56,3 & 1,35 & $1,03-1,77$ & \\
\hline \multicolumn{6}{|l|}{ Circunferência da cintura *** } \\
\hline Adequada & $520(67,9)$ & 39,8 & 1,00 & & $<0,001$ \\
\hline Aumentada & $130(17,0)$ & 48,8 & 1,23 & $0,99-1,51$ & \\
\hline Muito aumentada & $116(15,1)$ & 67,8 & 1,70 & $1,44-2,01$ & \\
\hline
\end{tabular}

IC95\%: intervalo de 95\% de confiança; IMC: índice de massa corporal; RP: razão de prevalência.

* Consumo médio de pelo menos uma vez no dia;

** Para a classificação do estado nutricional foi utilizado o IMC em kg/m², sendo considerado: baixo peso (< 18,5 adultos e $\leq$

22 idosos); eutrófico ( $\geq 18,5$ e $<25$ adultos, $>22$ e $<27$ idosos); sobrepeso ( $\geq 25$ e $<30$

adultos e $\geq 27$ idosos) e obesidade ( $\geq 30$, adultos) 29,30 ;

*** A circunferência da cintura adequada $(<94 \mathrm{~cm}$ para homens e $<80 \mathrm{~cm}$ para mulheres); aumentada $(\geq 94 \mathrm{~cm}$ para homens e $\geq 80 \mathrm{~cm}$ para mulheres) e muito aumentada ( $\geq 102 \mathrm{~cm} \mathrm{e} \geq 88 \mathrm{~cm}$ para homens e mulheres, respectivamente) 29 .

A maioria dos indivíduos consumia legumes/ verduras e frutas pelo menos uma vez no dia (Tabela 2), apenas $4,5 \%$ e $10,5 \%$ faziam o consumo por 3 ou mais vezes no dia, respectivamente. $\mathrm{O}$ não consumo de legumes/verduras apresentou uma relação positiva com hipertensão arterial (Tabela 2). Foi observado que 59,9\% dos indivíduos consumiam mais de $5 \mathrm{~g}$ de sal por dia, com o consumo médio de $8,0 \pm 6,3 \mathrm{~g}$. Contudo, diferença estatística para o sal foi observada apenas quando comparado o consumo médio de sal dos hipertensos e não hipertensos $(8,5 \pm 6,4 \mathrm{~g} v s$. $7,6 \pm 6,1 \mathrm{~g} ; \mathrm{p}<0,05$, respectivamente).

Cerca de $20 \%$ relataram ter problemas com o sono nos últimos 15 dias, com prevalência de hipertensão arterial 1,16 vez maior que os que não relataram ter dificuldades para dormir. Em relação ao estado nutricional, mais de $30 \%$ apresentaram excesso de peso, $6,4 \%$ foram considerados obesos, com prevalência de hipertensão 
arterial $49 \%$ maior quando comparados aos de baixo peso/eutróficos. A prevalência de hipertensão arterial foi $70 \%$ maior entre os que apresentaram CC muito aumentada em relação aos com CC adequada (Tabela 2). Observa-se neste estudo uma alta correlação entre IMC e CC ( $\mathrm{r}=$ 0,$72 ; \mathrm{p}<0,001$ ).

Para atividades esportivas ou artísticas em grupos nos últimos 12 meses, 72,6\% não realizaram estas atividades, enquanto que $14,5 \%$ participaram uma vez ou mais na semana. A inatividade física apresentou uma prevalência de $26,3 \%$. A hipertensão arterial foi associada à realização de atividades em grupo na direção esperada e assumiu a forma de um gradiente: quanto menor a frequência de realização de atividades maior a prevalência de hipertensão. Uma maior prevalência de hipertensão foi observada entre os indivíduos classificados como inativos (Figura 2).

$\mathrm{Na}$ análise multivariada hierárquica (Tabela 3), observa-se associação entre o entorno físico e social e a hipertensão, na qual os indivíduos que relataram não se sentirem seguros ao caminhar na vizinhança durante o dia e a noite apresentaram maior prevalência de hipertensão arterial. No modelo 2, a hipertensão arterial foi associada com a idade, classe econômica e escolaridade. A inatividade física foi a única variável do bloco de estilo de vida que apresentou associação com a hipertensão arterial (modelo 3). Com a inclusão da variável IMC (modelo 4), determinante próximo da hipertensão, foi observado que o excesso de peso (sobrepeso e obesidade) manteve-se associado à hipertensão.

\section{Discussão}

Entre quilombolas, uma população étnico-racial com características de grande exclusão histórico-social, a prevalência de hipertensão foi considerada elevada, principalmente quando comparada com estudos realizados em populações com faixas etárias semelhantes e que utilizaram a mesma definição para hipertensão 6,15,32,33,34,35.

O presente estudo permitiu que fossem explorados os fatores associados à hipertensão arterial em comunidades quilombolas, controlando-se possíveis confusões destas associações. A hipertensão arterial mostrou-se associada ao fator distal - segurança na vizinhança; fatores intermediários - idade, classe econômica, escolaridade e inatividade física; e ao fator proximal - IMC.

Em concordância com estudos anteriores 32,33,34,35 a idade apresentou associação positiva com a hipertensão arterial, mesmo após ajustes por outras variáveis. É sabido que alterações próprias do envelhecimento como enrijecimento de artérias contribuem com a elevação da pressão arterial em indivíduos com mais de 60 anos 36 , contudo, no presente trabalho também foram constatados elevados valores de pressão arterial na população economicamente ativa, revelan-

Figura 2

Prevalência de hipertensão arterial em relação à realização de atividades em grupo nos últimos 12 meses e à inatividade física. Comunidades quilombolas de Vitória da Conquista, Bahia, Brasil, 2011.
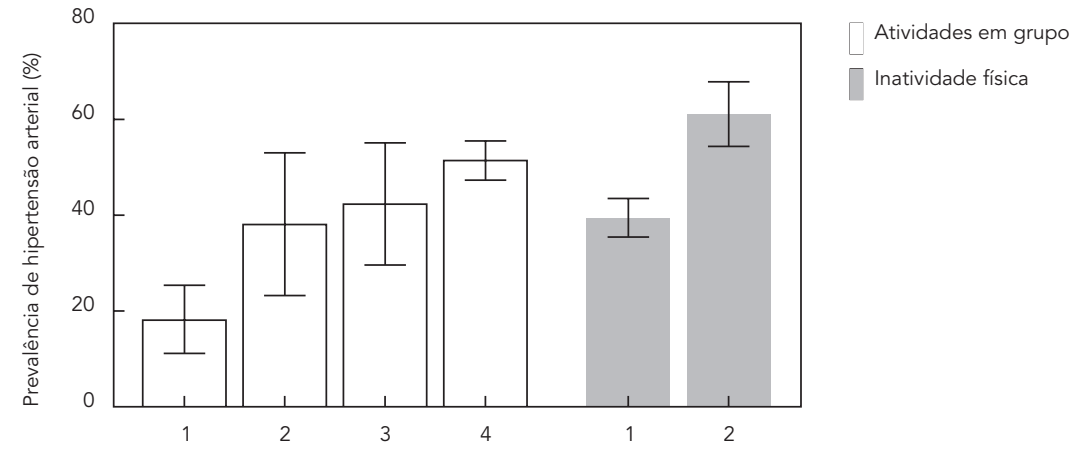

\footnotetext{
Realização de atividades em grupo nos últimos 12 meses $(p<0,001): 1$ = uma vez ou mais na semana; $2=$ uma vez ou mais no mês; 3 = uma vez ou mais no ano; 4 = nenhuma vez. Inatividade física $(p<0,001)$ : $1=$ não; $2=\operatorname{sim}$.
} 
Análise multivariada por meio da regressão de Poisson para hipertensão arterial e fatores associados. Projeto COMQUISTA, Brasil, 2011.

\begin{tabular}{|c|c|c|c|c|c|c|c|c|}
\hline \multirow[t]{2}{*}{ Variáveis } & \multicolumn{2}{|c|}{ Modelo 1} & \multicolumn{2}{|c|}{ Modelo 2} & \multicolumn{2}{|c|}{ Modelo 3} & \multicolumn{2}{|c|}{ Modelo 4} \\
\hline & RP & IC95\% & RP & IC95\% & RP & IC95\% & $\mathrm{RP}$ & IC95\% \\
\hline \multicolumn{9}{|l|}{ Segurança na vizinhança } \\
\hline Sim & 1,00 & & 1,00 & & 1,00 & & 1,00 & \\
\hline Não & 1,22 & $1,05-1,43$ * & 1,07 & $0,93-1,23$ & 1,08 & $0,94-1,25$ & 1,11 & $0,97-1,29$ \\
\hline \multicolumn{9}{|l|}{ Bloco 2: sociodemográfico } \\
\hline \multicolumn{9}{|l|}{ Idade (anos) } \\
\hline $18-29$ & & & 1,00 & & 1,00 & & 1,00 & \\
\hline $30-39$ & & & 1,63 & $1,08-2,48 * \star$ & 1,64 & $1,08-2,49 * *$ & 1,59 & $1,04-2,43 * \star \star$ \\
\hline $40-49$ & & & 2,32 & 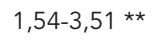 & 2,34 & $1,55-3,54 * \star$ & 2,19 & $1,44-3,32$ ** \\
\hline $50-59$ & & & 3,16 & $2,11-4,72 * \star$ & 3,23 & $2,16-4,84 * *$ & 3,08 & $2,05-4,63$ ** \\
\hline \multicolumn{9}{|l|}{ Escolaridade (anos) } \\
\hline 5 ou mais & & & 1,00 & & 1,00 & & 1,00 & \\
\hline $1-4$ & & & 1,73 & $1,21-2,49 * \star \star$ & 1,68 & $1,17-2,41 * \star \star$ & 1,71 & $1,19-2,46 * \star \star$ \\
\hline Nunca estudou & & & 1,75 & $1,23-2,48$ *** & 1,71 & $1,21-2,43$ *** & 1,69 & $1,19-2,40$ *** \\
\hline \multicolumn{9}{|l|}{ Classe econômica } \\
\hline $\mathrm{C}$ e B2 & & & 1,00 & & 1,00 & & 1,00 & \\
\hline D & & & 1,046 & $0,80-1,33$ & 1,05 & $0,84-1,32$ & 1,08 & $0,86-1,37$ \\
\hline$E$ & & & 1,38 & $1,10-1,74^{\star \star \star}$ & 1,36 & $1,08-1,71 * \star \star$ & 1,49 & $1,17-1,90 * \star \star$ \\
\hline \multicolumn{9}{|l|}{ Bloco 3: estilo de vida } \\
\hline \multicolumn{9}{|l|}{ Inatividade física } \\
\hline Não & & & & & 1,00 & & 1,00 & \\
\hline Sim & & & & & 1,19 & $1,02-1,38$ * & 1,16 & $1,00-1,36$ \\
\hline \multicolumn{9}{|c|}{ Bloco 4: índices antropométricos } \\
\hline Baixo peso/Eutrófico & & & & & & & 1,00 & \\
\hline Sobrepeso & & & & & & & 1,22 & $1,05-1,42$ * \\
\hline Obeso & & & & & & & 1,78 & $1,33-2,37$ ** \\
\hline Critério Akaike & & 71,6 & & 62,30 & & 48,00 & & 114,20 \\
\hline
\end{tabular}

IC95\%: intervalo de 95\% de confiança; IMC: índice de massa corporal; RP: razão de prevalência.

Modelo 1: Ajustado entre as variáveis do bloco entorno físico e social; Modelo 2: Ajustado entre as variáveis dos blocos entorno físico e social e

sociodemográfico; Modelo 3: Ajustado entre as variáveis dos blocos entorno físico e social, sociodemográfico e estilo de vida; Modelo 4: Ajustado entre as variáveis dos blocos entorno físico e social, sociodemográfico, estilo de vida e índices antropométricos.

${ }^{*} \mathrm{p}<0,05$

$\star * \quad p<0,001$

$\star \star \star ~ p<0,01 ;$

\# IMC em kg/m², sendo considerado: baixo peso ( $<18,5$ adultos e $\leq 22$ idosos); eutrófico ( $\geq 18,5$ e $<25$ adultos $>22$ e $<27$ idosos); sobrepeso $(\geq 25$ e $<30$ adultos, $\geq 27$ idosos) e obesidade ( $\geq 30$ adultos) 29,30

do a magnitude da hipertensão arterial entre os quilombolas, chamando a atenção para possíveis repercussões cardiovasculares provenientes deste achado.

As condições de vulnerabilidade das comunidades quilombolas estudadas foram observadas pelos baixos níveis de escolaridade e pela concentração em classes econômicas mais baixas, podendo estes fatores parcialmente justificar a elevada prevalência de hipertensão arterial encontrada. O impacto nas condições de saúde de populações que se concentram em níveis socioeconômicos mais baixos é decorrente da falta de acesso ao sistema de saúde, grau de informação, compreensão do problema e adesão ao tratamento 37 . Estudos indicam que a hipertensão 
arterial está mais relacionada ao estilo de vida do que a diferenças genéticas entre raças; a elevada prevalência de hipertensão arterial observada em populações africanas em relação às de origem europeia foi atenuada após ajuste por status socioeconômico 38 .

Em relação às variáveis mais distais (entorno físico e social), segurança na vizinhança apresentou associação positiva com hipertensão arterial. Portanto, é plausível a discussão de que situações de insegurança podem contribuir com o aumento da prevalência de hipertensão arterial, via inatividade física e estresse psicossocial 39,40. Indivíduos que se sentem inseguros em caminhar na vizinhança, além de estarem em situação de estresse, apresentam menor chance de realizar atividade física ou atividades relacionadas ao lazer 39 .

A associação observada entre fumo e hipertensão deixou de ser significativa após ajuste no modelo multivariado. Os resultados que relacionam hipertensão arterial e fumo em estudos transversais são controversos, alguns encontraram associações em análise bivariada como o presente estudo 32,33 e outros não observaram associações significativas 15,35.

Tem sido relatado na literatura o consumo de álcool como um fator de risco para doenças cardiovasculares como a hipertensão, principalmente em estudos longitudinais 41 . No presente trabalho foi encontrado um resultado contrário ao esperado; a prevalência de hipertensão arterial foi menor no grupo exposto. Ao estratificar essa associação pela hipertensão autorreferida (dados não mostrados) foi observado que a maioria dos indivíduos que sabiam que eram hipertensos relatou menor consumo de álcool. Essa causalidade reversa entre álcool e hipertensão arterial tem sido demonstrada em estudos transversais 35 . Se por um lado indivíduos previamente diagnosticados e com informações sobre a hipertensão arterial poderiam procurar mais fatores de proteção do que aqueles que desconhecem a doença, por outro, podem fornecer respostas inverídicas em entrevistas por conhecerem os prejuízos causados pelo álcool.

Quanto aos hábitos alimentares da população quilombola, uma pequena parcela atendeu à recomendação 27 de consumir no mínimo 3 vezes ao dia frutas e verduras. A escassez de locais para adquirir esses alimentos destacou-se como uma grande barreira de acesso, uma vez que 92,6\% dos indivíduos que não faziam consumo de verduras relataram não existir local para comprar esses alimentos na vizinhança.

Maiores prevalências de hipertensão arterial foram observadas nos grupos quilombolas que relataram não consumir frutas e verduras/ legumes, porém tal associação também não se manteve no modelo multivariado. Contudo, são conhecidos os benefícios da alimentação saudável na prevenção de doenças crônicas não transmissíveis; o consumo insuficiente de frutas e verduras é responsável por 2,7 milhões de óbitos por ano ${ }^{42}$. A hipertensão é a doença crônica não transmissível mais frequente, sendo que as doenças cardiovasculares ainda são a principal causa de morte no Brasil 43, observando que a alimentação inadequada contribui diretamente para o aumento da incidência de hipertensão arterial 44.

A média do consumo de sal pelos quilombolas foi considerada elevada em relação à recomendada para a população brasileira ( $5 \mathrm{~g} /$ dia) 27 , sendo maior do que a encontrada na população

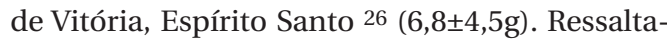
se que o alto consumo de sal dessa população poderia estar subestimado, uma vez que foi verificado apenas o sal de adição (utilizado no preparo dos alimentos) não sendo quantificado o consumo de sódio em alimentos industrializados e in natura. Mesmo que a associação entre sal e hipertensão arterial não tenha permanecido após ajustamento, a prática de redução do sal da alimentação deve ser incentivada nessa população, que apresentou um consumo elevado, uma vez que é sabido que a diminuição do sal na dieta contribui para reduzir os níveis de pressão arterial 45.

Assim como em estudos realizados em outras populações $6,15,35$, o excesso de peso foi um dos principais fatores associados à hipertensão arterial. Tal achado confirma a importante associação do excesso de peso e o impacto negativo de hábitos e comportamentos inadequados na saúde dos quilombolas. Semelhante ao trabalho realizado por Barbosa et al. ${ }^{46}$, e possivelmente devido à alta correlação encontrada, a CC perdeu o poder explicativo após ajuste por demais variáveis, permanecendo somente o IMC no modelo final.

A prevalência de inatividade física neste estudo foi menor do que a da população urbana de Pelotas, Rio Grande do Sul 28 (41,1\%) e de Belo Horizonte, Minas Gerais 47 (56,7\%). Contudo, foi maior do que a da população da zona rural do Vale do Jequitinhonha, Minas Gerais 48 (13,5\%). Indivíduos considerados inativos apresentam maiores chances de desenvolver doenças crônicas não transmissíveis 28 , como hipertensão arterial 47. O nível socioeconômico destaca-se entre os vários fatores que podem contribuir com o hábito de não realizar atividade física 49 , principalmente aquelas relacionadas ao lazer 47,48 . A falta de dinheiro, de informação e de ambientes favoráveis podem influenciar na ocorrência de 
inatividade física em populações economicamente desfavorecidas 49. Essa mesma linha de raciocínio pode ser utilizada para compreender a baixa prevalência de realização de atividades em grupos (artísticas e esportivas) observada neste estudo.

Dados do recente relatório sobre estatísticas sanitárias da Organização Mundial da Saúde (OMS) 50 revelaram que a população obesa do planeta aumentou 100\% entre 1980 e 2008, como reflexo das transformações nos padrões de produção e consumo de alimentos, assim como do estilo de vida sedentário. A presença de duas doenças crônicas não transmissíveis, como a hipertensão arterial e a obesidade, e a não realização de atividade física aumentam o risco de morbimortalidade por doenças cardiovasculares 50 .

Estudos realizados no Brasil demonstram a existência de desigualdades sociais nas prevalências de doenças crônicas não transmissíveis, inclusive na hipertensão, por ser a mais prevalente entre elas ${ }^{8}$. No presente trabalho, a hipertensão arterial apresentou um gradiente social que cresce na direção dos segmentos socialmente mais desfavorecidos, corroborando as condições de vulnerabilidade em que a população quilombola está inserida 16,17,19.

As posições socioeconômicas assumidas pelos indivíduos, bem como o estilo de vida, podem ser influenciados pelo contexto em que as populações vivem 24,51 . Fatores individuais e fatores contextuais podem determinar a hipertensão arterial em uma dada população 51 . Os entornos físicos e padrões sociais limitam o acesso a agentes condutores (locais para comprar frutas e verduras, área de lazer) de um estilo de vida saudável e podem resultar em hábitos e comportamentos inadequados (inatividade física, alimentação inadequada, excesso de peso). Torna-se evidente a importância de pesquisas que avaliem esses fatores como determinantes de um evento em saúde.

Dado ao delineamento, o estudo apresenta limitações quanto às inferências causais, relacionadas a problemas de temporalidade, como a possível causalidade reversa encontrada com o álcool, conflitando com trabalhos prévios. Quanto às perdas diferenciais, mesmo não sendo observadas diferenças significativas nas estimativas de ponto da prevalência de hipertensão, após a ponderação, não se pode descartar totalmente a possibilidade de um viés de seleção. Ademais, estudos para verificar o efeito do contexto em eventos de saúde devem ser delineados como tal, merecendo tamanho e complexidade amostral, além de análises multiníveis capazes de lidar com efeitos possíveis de agrupamentos entre as características dos indivíduos e seus contextos.

Apesar das limitações citadas, este foi o primeiro estudo de base populacional realizado em comunidades quilombolas do sudoeste da Bahia que avaliou a hipertensão arterial e fatores associados. Observa-se a escassez de trabalhos de base populacional nessas populações. Em dois estudos encontrados na literatura 52,53 que avaliaram a hipertensão arterial em comunidades quilombolas, a prevalência variou de $6,28 \% 52$ a $42 \% 53$, porém a comparabilidade com estes resultados torna-se prejudicada, uma vez que estes trabalhos apresentaram metodologias diferentes de um estudo de base populacional com delineamento amostral definido, como o presente trabalho.

Pesquisas em populações vulneráveis como os quilombolas são raras e podem ampliar os conhecimentos existentes sobre os determinantes da doença, no sentido de direcionar e contribuir para a implementação de políticas públicas de prevenção e controle, consoante com o plano de ações para enfrentamento das doenças crônicas não transmissíveis 54, lançado em 2011. Reforçam, portanto, a necessidade de ações intersetoriais, com a participação de órgãos da educação, desenvolvimento social, desenvolvimento agrário e cultura, criando oportunidades e estimulando a agricultura familiar para o aumento da produção e da oferta de alimentos in natura, com vistas à alimentação saudável. $\mathrm{O}$ aumento da prática de atividade física no combate ao excesso de peso sugere que elementos culturais, tais como danças e capoeira, praticados por esse grupo populacional podem funcionar como estímulo de atividades de lazer, potencializados pela criação de locais como facilitadores desta ação. Assim, a alta prevalência de hipertensão arterial encontrada reforça a necessidade de um amplo programa de promoção e acesso aos serviços de saúde para diagnóstico precoce, bem como orientações para o manejo adequado da mesma. 


\section{Resumen}

El objetivo de este estudio fue estimar la prevalencia de hipertensión arterial de residentes de las comunidades quilombolas y evaluar sus factores asociados. Es un estudio basado en la población, con una muestra de 797 individuos de 18 años o más. La hipertensión arterial se define como una presión arterial sistólica $\geq 140 \mathrm{mmHg}$ y/o presión arterial diastólica $\geq 90 \mathrm{mmHg}$ y/o el uso de medicamentos antihipertensivos. Se utilizó la regresión de Poisson con varianza robusta, con una entrada jerárquica de variables. La prevalencia de hipertensión arterial fue de 45,4\% (95\%IC: 41,89-48,85), los factores distales: la seguridad en el barrio, factores intermedios: edad, clase socioeconómica, la educación y la falta de actividad física, factores proximales como el índice de masa corporal estaban asociados con la hipertensión arterial. Existe la necesidad de promover la salud mediante la atención inclusiva de los quilombolas a través de acciones a nivel individual y poblacional. La alta prevalencia de la hipertensión arterial aumenta la necesidad de acceso a los servicios de salud para la prevención, el diagnóstico precoz y las directrices para una gestión adecuada.

Hipertensión; Factores de Riesgo; Comunidades Vulnerables; Grupo de Ascendencia Continental Africana; Estudios Transversales

\section{Colaboradores}

V. M. Bezerra contribuiu no desenvolvimento do projeto, revisão da literatura, análise dos dados e redação do artigo. A. C. S. Andrade colaborou na revisão da literatura, análise dos dados e revisão da versão final do artigo. C. C. César colaborou na análise dos dados e revisão da versão final do artigo. W. T. Caiaffa contribuiu no desenvolvimento do projeto, revisão da literatura, análise dos dados e redação do artigo.

\section{Agradecimentos}

A todos os pesquisadores do Projeto COMQUISTA pela participação na elaboração do projeto, planejamento e supervisão da coleta dos dados. À CAPES pelo financiamento do Doutorado Interinstitucional (DINTER), por meio do Edital 05/2009, Ação Novas Fronteiras. Ao CNPq pela bolsa produtividade em pesquisa da pesquisadora W. T. Caiaffa.

\section{Referências}

1. World Health Organization. Global health risks: mortality and burden of disease attributable to selected major risks. Geneva: World Health Organization; 2009.

2. Williams B. The year in hypertension. J Am Coll Cardiol 2008; 51:1803-17.

3. Olatunbosun ST, Kaufman JS, Cooperand RS, Bella AF. Hypertension in a black population: prevalence and biosocial determinants of high blood pressure in a group of urban nigerians. J Hum Hypertens 2000; 14:249-57.

4. Secretaria de Políticas de Saúde, Ministério da Saúde. Manual de doenças mais importantes, por razões étnicas, na população brasileira afro-descendente. Brasília: Ministério da Saúde; 2001. (Série A. Normas e Manuais Técnicos, 123).
5. Matos AC, Ladeia AM. Assessment of cardiovascular risk factors in a rural community in the Brazilian State of Bahia. Arq Bras Cardiol 2003; 81: 297-302.

6. Sarno F, Monteiro CA. Importância relativa do índice de massa corporal e da circunferência abdominal na predição da hipertensão arterial. Rev Saúde Pública 2007; 41:788-96.

7. Schmidt MI, Duncan BB, Hoffmann JF, Moura L, Malta DC, Carvalho RMSV. Prevalência de diabetes e hipertensão no Brasil baseada em inquérito de morbidade auto-referida, Brasil, 2006. Rev Saúde Pública 2009; 43:74-82. 
8. Barros MBA, Francisco PMSB, Zanchetta LM, César CLG. Tendências das desigualdades sociais e demográficas na prevalência de doenças crônicas no Brasil, PNAD: 2003- 2008. Ciênc Saúde Coletiva 2011; 16:3755-68.

9. Sociedade Brasileira de Cardiologia; Sociedade Brasileira de Hipertensão; Sociedade Brasileira de Nefrologia. VI diretrizes brasileiras de hipertensão. Arq Bras Cardiol 2010; 95(1 Suppl 1):1-51.

10. Moreira GC, Cipullo JP, Martin JF, Ciorlia LA, Godoy MR, Cesarino CB, et al. Evaluation of the awareness, control and cost-effectiveness of hypertension treatment in a Brazilian city: populational study. J Hypertens 2009; 27:1900-7.

11. Pereira MR, Coutinho MSSA, Freitas PF, D'Orsi E, Bernardi A, Hass R. Prevalência, conhecimento, tratamento e controle de hipertensão arterial sistêmica na população adulta urbana de Tubarão, Santa Catarina, Brasil, em 2003. Cad Saúde Pública 2007; 23:2363-74.

12. Instituto Brasileiro de Geografia e Estatística. Censo demográfico 2010. Características da população e dos domicílios. Resultados do universo. Rio de Janeiro: Instituto Brasileiro de Geografia e Estatística; 2011.

13. Batista LE, Volochko A, Ferreira CEC, Martins V. Mortalidade da população negra adulta no Brasil. In: Fundação Nacional de Saúde, organizador. Saúde da população negra no Brasil: contribuições para a promoção da equidade. Brasília: Fundação Nacional de Saúde; 2005. p. 237-314.

14. Lessa I. Epidemiologia da hipertensão arterial sistêmica e insuficiência cardíaca no Brasil. Rev Bras Hipertens 2001; 8:383-92.

15. Lessa I, Magalhães L, Araújo MJ, Almeida Filho N, Aquino E, Oliveira MMC. Hipertensão arterial na população adulta de Salvador (BA) - Brasil. Arq Bras Cardiol 2006; 87:747-56.

16. Silva JAN. Condições sanitárias e de saúde em caiana dos crioulos, uma comunidade quilombola do Estado da Paraíba. Saúde Soc 2007; 16:111-24.

17. Freitas DA, Caballero AD, Marques AS, Hernández CIV, Antunes SLNO. Saúde e comunidades quilombolas: uma revisão da literatura. Rev CEFAC 2011; 13:937-43.

18. Casa Civil, Presidência da República. Decreto no 4887, de 20 de novembro de 2003. Regulamenta o procedimento para identificação, reconhecimento, delimitação, demarcação e titulação das terras ocupadas por remanescentes das comunidades dos quilombos de que trata o art. 68 do Ato das Disposições Constitucionais Transitórias. http://www.pla nalto.gov.br/ccivil_03/decreto/2003/d4887.htm (acessado em 29/Mai/ 2012).

19. Calheiros FP, Stadtler HHC. Identidade étnica e poder: os quilombos nas políticas públicas brasileiras. Rev Katál 2010; 13:133-9.

20. Universidade Federal da Bahia. Relatório técnico do projeto COMQUISTA - comunidades quilombolas de Vitória da Conquista: avaliação de saúde e seus condicionantes, 2011. http://projetocom quista.wordpress.com (acessado em 05/Jun/2012).

21. Pesquisa Nacional de Saúde. Inquérito Região Integrada do Distrito Federal (RIDE/DF). http://www. pns.icict.fiocruz.br (acessado em 01/Abr/2011).
22. Coleman A, Freeman P, Steel S, Shennan A. Validation of the Omron MX3 Plus oscillometric blood pressure monitoring device according to the European Society of Hypertension international protocol. Blood Press Monit 2005; 10:165-8.

23. Chobanian AV, Bakris GL, Black HR, Cushman WC, Green LA, Izzo Jr. JL, et al. The seventh report of the Joint National Committee on Prevention, Detection, Evaluation, and Treatment of High Blood Pressure: the JNC 7 report. JAMA 2003; 289: 2560-71.

24. Diez Roux AV. Residential environments and cardiovascular risk. J Urban Health 2003; 80:569-89.

25. Saunders JB, Aasland OG, Babor TF, de la Fuente JR, Grant M. Development of the Alcohol Use Disorders Identification Test (AUDIT): WHO collaborative project on early detection of persons with harmful alcohol consumption II. Addiction 1993; 88:791-804.

26. Molina MCB, Cunha RS, Herkenhoff LF, Mill JG. Hipertensão arterial e consumo de sal em população urbana. Rev Saúde Pública 2003; 37:743-50.

27. Coordenação-Geral da Política de Alimentação e Nutrição, Secretaria de Atenção à Saúde, Ministério da Saúde. Guia alimentar para a população brasileira: promovendo a alimentação saudável. Brasília: Ministério da Saúde; 2008.

28. Hallal PC, Victora CG, Wells JCK, Lima RC. Physical inactivity: prevalence and associated variables in Brazilian adults. Med Sci Sports Exerc 2003;35:1894-900.

29. WHO Consulation on Obesity. Obesity: preventing and managing the global epidemic. Geneva: World Health Organization; 2000. (WHO Technical Report Series, 894).

30. Lipschitz DA. Screening for nutritional status in the elderly. Prim Care 1994; 21:55-67.

31. Victora CG, Huttly SR, Fuchs SC, Olinto MTA. The role of conceptual frameworks in epidemiological analysis: a hierarchical approach. Int J Epidemiol 1997; 26:224-7.

32. Hartmann M, Dias-da-Costa JS, Olinto MTA, Pattussi MP, Tramontini A. Prevalência de hipertensão arterial sistêmica e fatores associados: um estudo de base populacional em mulheres no Sul do Brasil. Cad Saúde Pública 2007; 23:1857-66.

33. Costa JSD, Barcellos FC, Sclowitz ML, Sclowitz IKT, Castanheira M, Olinto MTA, et al. Prevalência de hipertensão arterial em adultos e fatores associados: um estudo de base populacional urbana em Pelotas, Rio Grande do Sul, Brasil. Arq Bras Cardiol 2007; 88:59-65.

34. Cesarino CB, Cipullo JP, Martin JFV, Ciorlia LA, Godoy MRP, Cordeiro JA, et al. Prevalência e fatores sociodemográficos em hipertensos de São José do Rio Preto - SP. Arq Bras Cardiol 2008; 91:31-5.

35. Rosário TM, Scala LCN, França GVA, Pereira MR, Jardim PCBV. Fatores associados à hipertensão arterial sistêmica em Nobres-MT. Rev Bras Epidemiol 2009; 12:248-57.

36. Nichols WW, Orourke MF. McDonald's blood flow in arteries: theoretical, experimental, and clinical principles. 4th Ed. London: Edward Arnold; 1998. 
37. Ong KL, Cheung BMY, Man YB, Lau CP, Lam KSL. Prevalence, awareness, treatment, and control of hypertension among United States adults 19992004. Hypertension 2007; 49:69-75.

38. Agyemang C, Addo J, Bhopal R, Aikins Ade G, Stronks K. Cardiovascular disease, diabetes and established risk factors among populations of subSaharan African descent in Europe: a literature review. Global Health 2009; 5:7.

39. Centers for Disease Control. Neighborhood safety and the prevalence of physical inactivity - selected states, 1996. MMWR Morb Mortal Wkly Rep 1999; 48:143-6.

40. Lima Jr. E, Lima Neto E. Hipertensão arterial: aspectos comportamentais - estresse e migração. Rev Bras Hipertens 2010; 17:210-25.

41. O'Keefe JH, Bybee KA, Lavie CJ. Alcohol and cardiovascular health. J Am Coll Cardiol 2007; 50:1009-14.

42. Organização Pan-Americana da Saúde; Organização Mundial da Saúde. Prevenção de doenças crônicas, um investimento vital. Geneva: Organização Mundial da Saúde; 2005.

43. Schmidt MI, Duncan BB, Silva GA, Menezes AM, Monteiro CA, Barreto SM, et al. Chronic non-communicable diseases in Brazil: burden and current challenges. Lancet 2011; 377:1949-61.

44. Forman JP, Stampfer MJ, Curhan GC. Diet and lifestyle risk factors associated with incident hypertension in women. JAMA 2009; 302:401-11.

45. Pimenta E, Gaddam KK, Oparil S, Aban I, Husain S, Dell'Italia LJ, et al. Effects of dietary sodium reduction on blood pressure in subjects with resistant hypertension results from a randomized trial. Hypertension 2009; 54:475-81.

46. Barbosa LS, Scala LCN, Ferreira MG. Associação entre marcadores antropométricos de adiposidade corporal e hipertensão arterial na população adulta de Cuiabá, Mato Grosso. Rev Bras Epidemiol 2009; 12:237-47.
47. Ferreira AD, César CC, Malta DC, Andrade ACS, Ramos CGC, Proietti FA, et al. Validade de estimativas obtidas por inquérito telefônico: comparação entre VIGITEL 2008 e Inquérito Saúde em Beagá. Rev Bras Epidemiol 2011; 14:16-30.

48. Bicalho PG, Hallal PC, Gazzinelli A, Knuth AG, Velásquez-Meléndez G. Atividade física e fatores associados em adultos de área rural em Minas $\mathrm{Ge}$ rais, Brasil. Rev Saúde Pública 2010; 44:884-93.

49. Gidlow C, Johnston LH, Crone D, Ellis N, James D. A systematic review of the relationship between socio-economic position and physical activity. Health Educ J 2006; 65:338-67.

50. World Health Organization. World health statistics 2012: noncommunicable diseases: a major health challenge of the 21st century. Geneva: World Health Organization; 2012.

51. Mujahid MS, Diez Roux AV, Morenoff JD, Raghunathan TE, Cooper RS, Ni H, et al. Neighborhood characteristics and hypertension. Epidemiology 2008; 19:590-8.

52. Jardim PC, Carneiro O, Carneiro SB, Baiocchi MN Arterial blood pressure in the remaining isolated black community of a quilombo north of GoiásKalunga. Arq Bras Cardiol 1992; 58:289-93.

53. Mingroni-Neto RC. Doenças modernas nos antigos quilombos: a obesidade e a hipertensão no Vale do Ribeira-SP. In: Volochko A, Batista LE, organizadores. Saúde nos Quilombos. São Paulo: Instituto de Saúde; 2009. p. 179-91. (Temas em Saúde Coletiva, 9).

54. Ministério da Saúde. Plano de ações estratégias para enfrentamento das doenças crônicas não transmissíveis (DCNT) no Brasil, 2011-2022. http:// portal/saude/profissional/area.cfm?id_area $=1818$ (acessado em 16/Mai/2012).

Recebido em 15/Nov/2012

Versão final reapresentada em 16/Abr/2013

Aprovado em 24/Abr/2013 